\title{
Gardelle, Laure. Semantic Plurality: English Collective Nouns and Other Ways of Denoting Pluralities of Entities. John Benjamins, 2019. 215 pages Yolanda Fernández-Pena (yolanda.fernandez@uvigo.es) Universidade de Vigo
}

This volume provides an unprecedented, comprehensive account of and consistent gradient of construal for the various means of expressing lexical-level (bunch, clothes) and discourse-built (every car) semantic plurality in English, with a focus on the delimitation of the category of 'collective nouns.' Moving away from the traditional reliance on the variable agreement patterns of collective nouns to focus on their construal of the plurality, Gardelle's study proposes not only a consistent characterisation of this class of semantically plural nouns as wholes of highly integrated units but also accommodates, by comparison, looser ways of integrating units in a plurality such as 'aggregates' (furniture, these crew), which denote classes and whose starting point of the construal is the plurality, and 'groupings' (sheep, lots of students), where the units are more highly individuated, among others.

The book opens with an introduction which frames the study within a semantic feature approach, which enables the exploration of the expression of semantic plurality (i.e. "more than one," 1) at both the lexical and the discourse levels, thus escaping from the constraints imposed by grammatical number. In this initial chapter, Gardelle narrows down the concept of 'plurality,' whether external (i.e. morphosyntactic) or internal (i.e. a /plurality/ feature at lexical level), to pluralities with discrete entities. The reader is also informed of the leading questions of the research, which mostly hinge on the delimitation and characterisation of the category 'collective noun,' "the problematic backbone of references to pluralities" (13), as well as on its place among the various means of denoting pluralities in English. Taken as the pivotal category of Gardelle's study of pluralities, the chapter closes with a preliminary characterisation of collectives as nouns that (i) inherently denote an unprototypical meronymic (i.e. part/whole) relation, hence the term "collective wholes,' (ii) are construed as "grouping together a number of entities" (22) and (iii) contain an internal semantic/plurality/ feature. 
The second chapter of the volume tackles a recurrent topic and defining criterion in most investigations on collective nouns, namely their occurrence with singular and plural agreeing forms, the so-called 'hybrid agreement.' Gardelle, however, challenges previous literature by reconsidering the nature and motivations of hybrid agreement and demonstrating that it is not the most appropriate criterion to delimit the class of collective nouns because it is (i) imposed on their default agreement patterning as a result of the effect of the Animacy Hierarchy (i.e. licensed mostly by human collectives) (Croft 130) and their double layer of conceptualisation; (ii) constrained by the locality of the agreement domain according to the Agreement Hierarchy (Corbett) and (iii) not exclusive to collective nouns (e.g. gender systems). Gardelle finds further evidence of the unreliability of hybrid agreement to establish the boundaries of the class of collective nouns in a series of factors that are claimed to result in the opacification of number assignment: i.e. the asymmetry between plural (focus on individuals) and singular agreement (default pattern or focus on unity), together with regional, genre and stylistic variation. Most importantly, Gardelle also highlights the opacification caused by two factors which have attracted much of the recent scholarly attention on collective nouns: an overall diachronic shift towards singular agreement patterns with a great number of collective nouns since the nineteenth century, and the well-attested individual agreement preferences of collective nouns.

The main aim of Chapter 3 is to delimit the boundaries of the controversial category of collective nouns in English, which constitutes one of the main contributions of the study and has almost no precedents in the literature (Depraetere). To this end, Gardelle first describes the construal of the collective nouns that license hybrid agreement to then work outwards to accommodate the other means of denoting pluralities that are discussed in Chapters 4 to 6. To the defining features of collectives described in Chapter 1, she adds (i) 'cohesion of the units,' (ii) 'boundedness' of their plurality at lexical level through spatial (crowd), functional (team) or social (clergy) grouping, and (iii) acceptability (though with varying degrees of permeability) of 'non-additive properties' of size, age and shape that apply to the whole only, as a result of their double layer of conceptualisation (i.e. "a single whole, composed of units," 186). The 
chapter goes on to elaborate on the issue of hybrid agreement to provide further evidence of its unreliability as a defining feature of collective nouns, such as the fact that it is also licensed by an openended set of non-collective nouns (the hospital) which, according to Gardelle's criteria, are not collective at lexical level but "have collective reference due to a facet activated" through context-based metonymy at NP level (64). Against previous literature, Gardelle thus restricts 'collective noun' to count collective-only nouns (committee) and nouns with a collective sense at lexical level (association), thus also excluding taxonomic uses (i.e. denoting classes) of nouns such as species and race. On this basis, and with data collected from the Oxford English Dictionary, she provides three unprecedented comprehensive lists of count collective nouns for human (population), animal (brood) and inanimate (flora) referents. As crucial as this contribution is the proposal of the 'organised plurality construction' (Det $+\mathrm{N} 1+o f+N 2$ s), with which Gardelle closes the debate over the fuzzy boundary between literal, metaphorical and quantificational uses of collective nouns by accommodating them all (apart from other non-collective $\mathrm{N} 1 \mathrm{~s})$ in the construction and attributing their differences to the disactivation of the /group/ seme and/or their (semantic and/or syntactic) reanalysis as quantificational idioms.

Discourse-acquired plurality and its construal are the main focus of Chapter 4. None of the four means of expressing plurality that Gardelle surveys is claimed to be 'collective,' but the discussion is intended as a point of reference from which to integrate non-count nouns denoting pluralities (Chapters 5 and 6). On the one hand, she examines the construal of pluralities with partly substantivised adjectives and count plural nouns, with which greater integration of the units is achieved. Gardelle presents the former in a gradient of nominalisation: from less (the English) to more (three injured, the accused) substantivised items, with plurality being construed at the NP level as an 'aggregate of non-countable or partially countable units', respectively. Full nominalisation (Americans), by contrast, results in higher individuation of the units ('grouping of fully differentiable units'). As regards the plurality expressed by count nouns, Gardelle claims that it "does not just denote a sum [but] a construal of its own" (106), as a 'grouping' that may differ in the individuation of the units: while morphologically marked plural nouns (kids, children) and plural 
nouns with an obligatory zero morpheme (sheep) denote 'groupings of fully differentiable entities', when the zero marker is optional, as in three elephant, the entities are backgrounded further and less loosely integrated as 'non-differentiable.' A different case is that of uninflected plurals which Gardelle demonstrates result from type coercion from collective nouns (these crew): they construe the plurality with a lower degree of integration of the units in an 'aggregate of partially countable entities', with the plural noun often serving as a hyperonym of plural classes (i.e. crew $=$ cooks + stewards + sailors). On the other hand, the author reflects upon the construal of the discourse-acquired semantic plurality with the lowest degree of integration of the units: i.e. conjoined noun phrases (John and Mary, neither John nor Mary), which are claimed to still exhibit cohesion of the units despite a greater degree of individuation of the entities ('set of loosely connected elements'), and the borderline case of 'bound variable singularity' (eachlevery student), where plurality is only indirectly expressed and inferred from the "mental representation [...] of one unit at a time" (131).

Chapter 5 contributes significantly to delimiting the boundary of collective nouns by excluding non-count singular nouns that express semantic plurality, particularly the so-called 'furniture nouns,' which have long been a matter of debate in the literature of collective nouns. Unlike previous accounts, the broader perspective of Gardelle's study allows her to compare their construal against that of other means of denoting semantic plurality, thus contending that they are non-count and that their imposed lexical number has consequences for their construal: they tend to foreground the unity and license singular agreement patterns. This construal is in fact the key factor, Gardelle claims, to exclude them from 'collective nouns,' as they denote a nontaxonomic hyperonymic relation whereby the furniture noun serves as a superordinate term that groups plural classes (chairs, tables) on the grounds of their functional properties ('aggregate'). In this chapter, she also explores the construal of other non-count nouns that denote aggregates of homogeneous entities (foliage) and animals (livestock) and informs us of their non-collective status. Purported non-count nouns expressing pluralities of humans (management), however, are found to stand out from the rest of the nouns discussed for not being truly non-count and expressing collective reference, which grants a higher integration of the units in comparison. 
Chapter 6 is devoted to lexical plurals, which mainly express pluralities of inanimate entities, as in belongings and clothes. Most of the scholarly attention that these pluralities have attracted concurs that they are not collective nouns, owing to the lack of a mismatch between a singular morphology and a plural denotation. Gardelle reaches the same conclusion but explains their exclusion from 'collective nouns' on the grounds of their different construal. In her view, lexical plurals are non-count and denote pluralities of entities of different classes (i.e. belongings $=$ stuff, money, etc.), with their plurality being thus construed as an aggregate without the high individuation of the units of plural count nouns (children) but with a looser integration than with non-count singular nouns (furniture), owing to their marked plural morphology. The author also acknowledges the great instability of the plural number in this category, which results in the reanalysis of some lexical plurals as non-count singular (memorabilia) and count (groceries > grocery store) nouns. Despite this finding, her study also reveals that their different construal motivates their nonetheless frequent occurrence: unlike count plural nouns, lexical plurals denote pluralities of heterogeneous entities where the plurality and not the unity (vs non-count singular nouns; furniture) is foregrounded. The grammatical restrictions imposed by productive endings such as -ables and -ings in English (labelled 'morphological attractors') is adduced as the main explanation for the creation of new lexical plurals. As is the case with other uninflected lexical plurals coerced from collective nouns to denote 'groupings of fully differentiable entities,' such as people and folk, Gardelle also rejects the inclusion of cattle in the category 'collective' (cf. Depraetere), which is in turn put on a par with lexical plurals as a hyperonym (i.e. aggregate) of plural classes (cows, bulls).

The last chapter provides a brief summary of the main characteristics of the construals of the pluralities surveyed in Chapters 3 to 6 , which, broadly speaking, differ in their degree of integration of the entities in the plurality. It is on this basis that Gardelle proposes the so-called 'Scale of Unit Integration' (189), a highly consistent system which captures the differences between the highest degree of integration denoted by collective wholes (collection) and the progressively higher individuation of the units with aggregates 
(furniture, these crew), groupings (three elephant, cats) and, particularly, conjoined noun phrases (John and Mary) and bound variable singularity (each student). The book closes with a brief discussion on prospective lines of research such as a potential extension of the scale to inferences of plurality and the exploration of particulate masses (sand, grass) along a 'Scale of Part Integration.'

This review of the volume leaves little doubt of the importance of Gardelle's account of semantic plurality in English. In fact, the bulk of literature that she reviews throughout the book underscores the need of both a broader approach to the topic and an in-depth revision and redefinition of the boundaries of categories such as 'collective nouns' and '(semantically plural) non-count nouns' that grant a better understanding of the subject. In response to previous studies and previously unsolved debates, Gardelle provides consistent typologies and comprehensive descriptions of semantic pluralities which will undoubtedly benefit not only further research but also lexical databases, as she herself notes.

One of the major breakthroughs of the volume is the delimitation of the boundaries of the wayward category of collective nouns. Much of the research to date has focused on the characterisation of the variable agreement patterns of this class of nouns, based on a certain number of collective nouns often taken from the lists provided in reference grammars. However, the lack of consensus and homogeneous classification of these nouns in both grammars and empirical studies has left little room for broad generalisations beyond individual agreement preferences and has contributed little to shedding light on the categorisation of these nouns. The extensive list of collective nouns presented in this volume and the distinction between truly collective nouns (collection, host), context-based collective reference (the whole street) and the 'organised plurality construction' (a crowd of tourists) will certainly be of great help for future research to refine and improve the existent accounts with respect to, for instance, agreement variation.

The 'organised plurality construction' is in fact another proposal with far-reaching implications: not only does it account for a plurality denoted at construction level (a busyness of ferrets) but it also 
accommodates the literal (a crowd of people) and the extended uses of collective nouns in complex noun phrases, i.e. metaphorical (quantificational reading: a shoal of visitors) and quantificational idioms (truly non-collective: these couple of emails). In essence, the extensions of the literal use capture the natural path of grammaticalisation described under other frameworks for collective nouns such as bunch (i.e. a bunch more, buncha; Brems 188-191). Full reanalysis into a quantificational idiom is "rare" (97), Gardelle claims, an observation which I think is far from strange considering that the productivity, functionality and expressiveness of the quantifying use of complex collective noun phrases lies precisely in the conceptual and syntactic persistence of certain traits of their nominal properties (i.e. unitising meaning, modification). It remains to be seen, however, how the partitive construction (a bunch of the visitors) fits in with Gardelle's account. She only notes that this structure "denotes a set from which a portion, a subset, is extracted" (90), but its construal of the plurality seems worth exploring owing to the quantificational reading (relative to the plural N2) attributed to these structures (e.g. Koptjevskaja-Tamm).

To conclude, it is worth highlighting again the innovative approach of the research. The focus on the construal of the plurality of entities with collective nouns not only solves much-debated controversies around this category of nouns but also elegantly accounts for the fine distinctions among all of the pluralities surveyed in Chapters 3 to 6, at lexical and clausal level, as derived from the different contribution to the construal of their grammatical number and countability, together with some hyperonymic and coerced uses. Useful and informative introductory and concluding summaries of each section guide the reader towards the succinct but enlightening final chapter, which wraps up the discussion with the 'Scale of Unit Integration for semantic pluralities of units.' In a nutshell, Gardelle's book is a stimulating reading and insightful contribution to the study of pluralities and, in general, to the field of linguistics which puts an end to several long-standing debates, particularly on the concept and the category of collective nouns, a class of nouns which had been discussed widely in the literature to date but mainly in rather vague terms. 


\section{Note}

${ }^{1}$ The author of this review is grateful to the Regional Government of Galicia (grants no. ED481B-2019/077, ED431C 2017/50), the Spanish State Research Agency and the European Regional Development Fund (grant no. FFI2016-77018-P) for generous financial support.

\section{Works Cited}

Brems, Lieselotte. Layering of Size and Type Noun Constructions in English. Mouton de Gruyter, 2011.

Corbett, G. G. "The Agreement Hierarchy." Journal of Linguistics, vol. 15, no. 2, 1979, pp. 203-24.

Croft, William. Typology and Universals. Cambridge UP, 2003 (1990). Depraetere, Ilse. "On Verbal Concord with Collective Nouns in British English." English Language and Linguistics, vol. 7, no. 1, 2003, pp. 85-127.

Koptjevskaja-Tamm, Maria. “'A Lot of Grammar with a Good Portion of Lexicon': Towards a Typology of Partitive and PseudoPartitive Nominal Constructions." Form and Function in Language Research: Papers in Honour of Christian Lehmann, edited by Johannes Helmbrecht, Yoko Nishina, Yong-Min Shin, Stavros Skopeteas, and Elisabeth Verhoeven, Mouton de Gruyter, 2009, pp. 329-46.

Oxford English Dictionary, $3^{\text {rd }}$ edn. online. www.oed.com. 